\title{
ESPACES FIBRÉS ASSOCIÉS ET PRÉ-ASSOCIÉS
}

\author{
J. L. KOSZUL
}

Introduction. Dans la première partie de cet article, on généralise une construction donnée dans [2], conduisant à l'homologie des espaces classifiants de groupe $\Gamma$. Cette construction utilise au départ un espace fibré principal $Y$ de groupe $\Gamma$, le cas traité dans [2] étant celui où $Y=\Gamma$. Elle permet de définir dans le module de cohomologie de la base $X$ de $Y$ une filtration par une suite de sous-modules dont l'intersection est le module des classes caractéristiques. Dans la seconde partie, la filtration précédente est étendue à $H^{1}(X, \mathbf{G})$ où $\mathbf{G}$ est le faisceau des germes d'applications continues de $X$ dans un groupe topologique G. On obtient ainsi dans $H^{1}(X, \mathbf{G})$ des sous-ensembles $Q_{2} \subset Q_{1} \subset H^{1}(X, \mathbf{G})$. Cette classification s'étend au cas holomorphe. Les espaces fibrés principaux de base $X$ de groupe $\mathrm{G}$ correspondant à $Q_{2}$ sont les espaces fibrés de groupe $G$ associés à $Y$. Les espaces fibrés correspondant à $Q_{1}$ sont appelés des espaces fibrés pré-associés à $Y$. Ce sont encore des espaces fibrés trivialisés par la projection de $Y$ sur $X$. Pour cette raison, ils peuvent être définis par un facteur $k: Y \times \Gamma \rightarrow G$. Pour qu'un facteur $k$ corresponde à un espace fibré $P$ préassocié à $Y$, il faut et il suffit qu'il existe une application continue $g: Y^{2} \rightarrow G$ telle que

$$
g\left(y, y^{\prime}\right) k\left(y^{\prime}, s\right)=k(y, s) g\left(y s, y^{\prime} s\right)
$$

quels que soient $y, y^{\prime} \in Y$ et $s \in \Gamma$. Le choix d'une application $g$ vérifiant cette condition présente de grandes analogies avec le choix d'une connexion dans l'espace $P$. En fait, dans le cas différentiable, et pour un groupe $\Gamma$ discret, on montre que $g$ détermine une connexion dans $P$.

1. Complexes topologiques. Soit $T$ la catégorie préadditive dont les objets sont les espaces topologiques, le groupe $\operatorname{Hom}(X, Y)$ des homomorphismes d'un

Received March 6, 1959. 
espace $X$ dans un espace $Y$ étant le groupe abélien libre ayant pour base l'ensemble des applications continues de $X$ dans $Y$. La composition des homomorphismes est définie, par linéarité, à partir de la composition des applications. On passe de la manière habituelle de la catégorie $T$ à la catégorie $T(1)$ des complexes de $T$ ou complexes topologiques. Un complexe topologique $X_{*}$ est donc défini par une famille $\left(X_{p}\right)$ ( $p$ entier) d'espaces topologiques et par la donnée, pour tout entier $p$, d'un homomorphisme $d_{p} \in \operatorname{Hom}\left(X_{p}, X_{p-1}\right)$ tel que $d_{p-1} d_{p}=0$ pour tout $p$. Etant donnés deux complexes topologiques $X_{*}$ et $Y_{*}$, un homomorphisme $h_{*}$ de $X_{*}$ dans $Y_{*}$ est une famille d'homomorphisme $h_{p} \in \operatorname{Hom}\left(X_{p}, Y_{p}\right)$ vérifiant la condition $d_{p} h_{p}=h_{p-1} d_{p}$ pour tout $p$. Deux homomorphismes $h_{*}$ et $h_{*}^{\prime}$ de $X_{*}$ dans $Y_{*}$ sont homologues si il existe une famille d'homomorphismes $k_{p} \in \operatorname{Hom}\left(X_{p}, Y_{p+1}\right)$ tels que $d_{p+1} k_{p}+k_{p-1} d_{p}=h_{p}-h_{p}^{\prime}$ quel que soit $p$.

Pour tout espace topologique $X$, on notera $R(X)$ le complexe topologique défini par

$$
\begin{aligned}
& R_{p}(X)=X^{p+1} \quad \text { pour } p \geq 0, \quad R_{p}(X)=\phi \quad \text { pour } p<0 \\
& d_{p}=\sum_{i=0}^{i=p}(-1)^{i} d_{p, i} \quad \text { pour } p>0, \quad d_{p}=0 \quad \text { pour } p \leq 0
\end{aligned}
$$

où $d_{p, i}(i=0,1, \ldots, p)$ est l'application $\left(x_{0}, x_{1}, \ldots, x_{p}\right) \rightarrow\left(x_{0}, x_{1}, \ldots, \hat{x}_{i}\right.$, $\left.\ldots, x_{p}\right)$ de $R_{p}(X)$ dans $R_{p-1}(X)$. Toute application continue d'un espace $X$ dans un espace $Y$ définit de manière évidente un homomorphisme de $R(X)$ dans $R(Y)$.

Homologie singulière des complexes topologiques. Pour tout entier $p$, on désigne par $\sigma^{p}$ le simplexe euclidien type de dimension $p$ et par $d^{p}$ l'homomorphisme de $\sigma^{p}$ dans $\sigma^{p+1}$ somme alternée des applications canoniques de $\sigma^{p}$ sur les faces de $\sigma^{p+1}$. Etant donné un complexe topologique $X_{*}$, on appelle module des chaines singulières de type $(p, q)$ (à coefficients entiers) de $X_{*}$, le $Z$-module $S_{p}\left(X_{q}\right)=\operatorname{Hom}\left(\sigma^{p}, X_{q}\right)$. On appelle complexe des chaines singulières de $X_{*}$ le complexe double $S_{*}\left(X_{*}\right)$ somme directe de la famille des $S_{p}\left(X_{q}\right)$ avec les opérateurs bord $\partial^{\prime}$ et $\partial^{\prime \prime}$ définis par

$$
\begin{aligned}
& \partial^{\prime} c=(-1)^{p+q+1} c d^{p-1} \\
& \partial^{\prime \prime} c=d_{q} c
\end{aligned}
$$

pour tout $c \in S_{p}\left(X_{q}\right)$. L'opérateur bord total sera noté $\partial=\partial^{\prime}+\partial^{\prime \prime}$.

Pour tout anneau commutatif $A$, le complexe $S_{*}\left(X_{*}\right) \underset{Z}{\otimes} A$ est appelé le. 
complexe des chaines singulières de $X_{:}$it coefficients dans $A$ et se note $S_{*}\left(X_{*}, A\right)$. Le complexe Hom $\left.S_{*} S_{*}\left(X_{*}\right), A\right)$ est appelé le complexe des cochaínes singulières de $X_{*}$ à coefficients dans $A$ et se note $S^{*}\left(X_{*}, A\right)$. On définit les modules d'homologie (resp. de cohomologie) de $X_{*}$ à coefficients dans $A$ en posant $H_{p}\left(X_{*}, A\right)=H_{p}\left(S_{*}\left(X_{*}, A\right)\right)\left(\operatorname{resp} . H^{\hat{1}}\left(X_{*}, A\right)=H^{f}\left(S^{*}\left(X_{*}, A\right)\right)\right)$. Si $h_{\text {: }}$ est un homomorphisme du complexe topologique $X_{*}$ dans le complexe topologique $Y_{*}, h_{*}$ définit un homomorphisme du complexe $S_{k}\left(X_{*}\right)$ dans $S_{*}\left(Y_{*}\right)$ et par conséquent des homomorphismes $h_{p}: H_{p}\left(X_{*}, A\right) \rightarrow H_{p}\left(Y_{*}, A\right)$ et $h^{p}: H^{p}\left(Y_{*}, A\right)$ $\rightarrow H^{p}\left(X_{*}, A\right)$ pour tout entier $p$. Deux homomorphismes homologues définissent les mêmes homomorphismes pour les modules d'homologie et de cohomologie.

2. Complexes $R(X) / G$. Soient $G$ un groupe topologique et $X$ un espace où le groupe $G$ opère de manière continue à droite. On fait opérer $G$ à droite dans chaque espace $R_{f}(X)=X^{p+1}$ en posant $\left(x_{0}, x_{1}, \ldots, x_{p}\right) s=\left(x_{9} s, x_{1} s, \ldots\right.$, $\left.x_{p} s\right)$ pour tout $\left(x_{0}, x_{1}, \ldots, x_{p}\right) \in X^{p-1}$ et tout $s \in G$. Chaque application $d_{t, 1}$ : $X^{p+1} \rightarrow X^{p}$ commute avec les opérations de $G$ et définit, par passage aux quotients une application continue $\mathbf{d}_{p, i}: X^{p+1} / G \rightarrow X^{\phi} / G$. On définit un complexe topologique $R(X) / G$ en prenant pour espace d'indice $p$ l'espace $R_{i}(X) / G$ $=X^{p+1} / G$ et pour opérateur $\mathbf{d}_{p}: X^{p+1} / G \rightarrow X^{p} / G$ l'homomorphisme $\sum_{i=0}^{i-p}(-1)^{i} \mathbf{d}_{p, 1}$. Les projections canoniques $q_{p}: X^{p+1} \rightarrow X^{p+1} / G$ constituent un homomorphisme canonique $q_{*}$ du complexe $R(X)$ sur le complexe $R(X) / G$.

Soient $X$ et $Y$ deux espaces où le groupe $G$ opère continuement à droite et soit $f$ une application continue de $X$ dans $Y$ qui commute avec les opérations de $G$. Soit $f_{*}$ l'homomorphisme de $R(X)$ dans $R(Y)$ défini par $f$. Chaque application $f_{p}: X^{p+1} \rightarrow Y^{p+1}$ commute avec les opérations de $G$ et définit par passage aux quotients une application continue $\mathbf{f}_{p}: X^{p+1} / G \rightarrow Y^{p+1} / G$. Les applications $\mathbf{f}_{p}$ constituent un homomorphisme $\mathbf{f}_{*}$ du complexe $R(X) / G$ dans le complexe $R(Y) / G$.

THÉorÈme 1. Soient $X$ et $Y$ deux espaces topologiques où le groupe $G$ opère continuement $\grave{a}$ droite et soient $f$ et $g$ deux applications continues de $X$ dans $Y$ commutant avec les opérations de G. Les homomorphismes $\mathbf{f}_{*}$ et $\mathbf{g}_{*}$ de $R(X) / G$ dans $R(Y) / G$ sont homologues.

En effet, pour tout entier $p \geq 0$, soit $k_{p}$ l'homomorphisme de $X^{p+1}$ dans $Y^{p+2}$ défini par $k_{i},=\sum_{i=1}^{i=p} k_{l, i}$ où $k_{t, i}$ est l'application continue définie par 


$$
k_{p, i}\left(x_{0}, x_{1}, \ldots, x_{p}\right)=\left(f\left(x_{0}\right), f\left(x_{1}\right), \ldots, f\left(x_{i}\right), g\left(x_{i}\right), \ldots, g\left(x_{p}\right)\right) ;
$$

un calcul direct montre que $g_{p}-f_{p}=d_{p+1} k_{p}+k_{p-1} d_{p}$. Comme chaque application $k_{p, i}$ commute avec les opérations de $G$ dans $X^{p+1}$ et $Y^{p+2}$, l'homomorphisme $k_{p}$ définit un homomorphisme $\mathbf{k}_{p}: X^{p+1} / G \rightarrow Y^{p+2} / G$ et $\mathbf{g}_{p}-\mathbf{f}_{p}=\mathbf{d}_{p+1} \mathbf{k}_{p}+\mathbf{k}_{p-1} \mathbf{d}_{p}$, ce qui démontre le théorème.

Appliquant ce qui précède au cas où $G$ est réduit à son élément neutre, on voit que si $I$ est un espace réduit à un point, alors pour tout espace topologique $X$, l'application de $X$ sur $I$ définit un isomorphisme canonique de $H_{p}(R(X), A)$ sur $H_{p}(R(I), A)$ pour tout entier $p$. Comme d'autre part, $H_{p}(R(I), A)$ est visiblement canoniquement isomorphe à $H_{p}(I, A)$, on voit que pour tout espace topologique $X, H_{p}(R(X), A)=(0)$ pour $p \neq 0$ et $H_{0}(R(X), A)$ est canoniquement isomorphe à $A .{ }^{1}{ }^{1}$

THÉORÈme 2. Soit $X$ un espace fibré principal de groupe $G$. Les applications continues de $G$ dans $X$ qui commutent avec les opérations de $G$ (c'est à dire de la forme $s \rightarrow$ as avec $a \in X)$ définissent un isomorphisme canonique de $H_{*}(R(G) / G, A)$ sur $H_{*}(R(X) / G, A)$ et un isomorphisme canonique de $H_{*}(R(X) / G)$ sur $H_{*}(R(G) / G, A)$.

On se bornera à indiquer le principe de la démonstration qui utilise un "complexe topologique double" $D$ formé avec les espaces $\left(G^{p} \times X^{q}\right) / G$. On définit des homomorphismes de $D$ dans $R(G) / G$ et dans $R(X) / G$. On montre que ces homomorphismes donnent des isomorphismes pour les modules d'homologie. On vérifie enfin que l'isomorphisme $H_{*}(R(G) / G, A) \rightarrow H_{*}(R(X) / G, A)$ ainsi obtenu est l'homomorphisme défini par les applications $G \rightarrow X$ de la forme $s \rightarrow a s$.

On observera que, si $X$ est un espace fibré principal de groupe $G$, alors chaque espace $R_{p}(X)$ du complexe topologique $R(X)$ est un espace fibré principal de groupe $G$. D'autre part $R(X)$ est acyclique d'après ce qu'on a vu plus haut.

THÉORÈme 3. Si $X$ est un espace fibré principal de groupe $G$ et $C$ un espace fibré classifiant de groupe $G([4])$, alors $H_{*}(R(X) / G, A)$ est canoniquement isomorphe à $H_{*}(C, A)$ et $H^{*}(R(X) / G, A)$ est canoniquement isomorphe à $H^{*}(C, A)$.

\footnotetext{
1) C'est aussi un cas particulier du Théorème 1 de [2].
} 
Le Théorème est démontré dans [2] pour le cas $X=G$. Le cas général en résulte d'après le Théorème 2 .

Soit $X \times R(G)$ le complexe topologique dont l'espace d'indice $n$ est $X \times R_{n}(G)$ et où $d_{n}=\left(l_{\lambda}, d_{n}\right), l_{X}$ désignant lapplication identique de $X$ sur $X$. Les projections $b_{n}: X \times R_{n}(G) \rightarrow R_{n}(G)$ définissent un homomorphisme $b_{*}$ de $X \times R(G)$ dans $R(G)$. On définit d'autre part un homomorphisme $f_{:}$de $X \times R(G)$ dans $X$ (considéré comme complexe topologique) en posant $f_{n}=0$ pour $n \neq 0$ et $f_{0}(x, s)=x$ pour tout $x \in X$ et $s \in G=R_{0}(G)$. On a un homomorphisme $c_{*}$ de $X$ dans $R(X)$ défini par $c_{0}=l_{X}$ et $c_{n}=0$ pour $n \neq 0$. Enfin, pour tout $a \in X$, on désigne par $a_{*}$ l'homomorphisme de $R(G)$ dans $R(X)$ défini par l'application $s \rightarrow$ as de $G$ dans $X$. Le diagramme

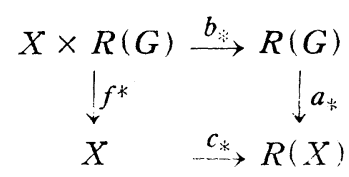

n'est pas commutatif. Cependant les homomorphismes $a_{*} b_{*}$ et $c_{*} f_{*}$ sont homologues. Pour tout entier $n \geq 0$, soit $k_{n}$ l'application de $X \times G^{n+1}$ dans $X^{n+2}$ définie par

$$
k_{n}\left(x, s_{0}, s_{1}, \ldots, s_{n}\right)=\left(x, a s_{0}, a s_{1}, \ldots, a s_{n}\right) .
$$

Pour tout $n$, on a $\mathrm{d}_{n+1} k_{n}+k_{n-1} d_{n}=a_{n} f_{n}-c_{n} b_{n}$. Les homomorphismes du diagramme (D) étant des combinaisons linéaires d'applications qui commutent avec les opérations de $G$, on déduit de (D) le diagramme:

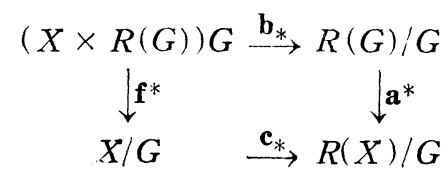

Comme les applications $k_{n}$ commutent avec les opérations de $G$, on voit que les homomorphismes $\mathbf{a}_{*} \mathbf{f}_{*}$ et $\mathbf{c}_{*} \mathbf{b}_{*}$ sont homologues. On a donc finalement, en cohomologie, un diagramme commutatif :

$\left(D^{\prime}\right)$

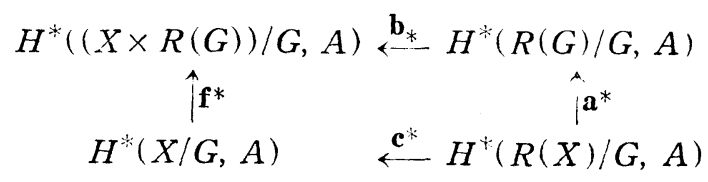

Il est démontré dans [2] que $\mathbf{f}^{*}$ est bijectif et que $\mathbf{f}^{*-1} \mathbf{b}^{*}$ est l'homomorphisme caractéristique de l'espace fibré principal $X$. Compte tenu du Théorème 2, la 
commutativité du diagramme $\left(\mathrm{D}^{\prime}\right)$ prouve le résultat suivant.

THÉORÈME 4. L'homomorphisme caractéristique de l'espace fibré principal $X$ est composé de l'isomorphisme $\mathbf{a}^{*-1}: H^{*}(R(G) / G, A) \rightarrow H^{*}(R(X) / G, A)$ et de l'homomorphisme canonique $\mathbf{c}^{*}: H^{*}(R(X) / G, A) \rightarrow H^{*}(X / G, A)$.

Soit $X$ un espace fibré principal de groupe $G$. Pour tout entier $n$, soit $K_{*}^{n}$ le complexe topologique

$$
X / G \leftarrow X^{2} / G \leftarrow \cdots \cdot X^{n+1} / G \leftarrow \emptyset \cdot \cdots
$$

qui s'obtient en tronquant le complexe $R(X) / G$. Il existe pour tout $n$ un homomorphisme canonique de $X / G$ dans $K_{*}^{n}$ défini par l'application identique de $X / G$ sur $K_{0}^{n}$. Cet homomorphisme définit pour tout $p$ un homomorphisme canonique de $H^{p}\left(K_{*}^{n}, A\right)$ dans $H^{p}(X / G, A)$ dont l'image sera notée $Q_{n}^{p}$. On obtient ainsi dans $H^{p}(X / G, A)$ une filtration:

$$
H^{p}(X / G, A)=Q_{0}^{p} \supset Q_{1}^{p} \subset \cdots Q_{p+1}^{p}=Q_{p+2}^{p}=\cdots=Q_{\infty}^{p}
$$

où $Q_{\infty}^{p}$ est le sous-module des classes caractéristiques de degré $p$. Pour tout $p>0$, le sous-module $Q_{1}^{p}$ est dans le noyau de l'homomorphisme de $H^{*}(X / G, A)$ dans $H^{*}(X, A)$ défini par la projection. En effet, l'image par cet homomorphisme d'une classe appartenant à $Q_{1}^{p}$ est une classe de cohomologie de $X$ à laquelle les projections $d_{1,0}$ et $d_{1,1}$ de $X^{2}$ sur $X$ font correspondre une même classe de cohomologie de $X^{2}$.

\section{II}

3. Espaces fibrés associés et pré-associés. Soit $Y$ un espace fibré principal de groupe $\Gamma$ et de base $X=Y / \Gamma$. Pour tout entier $n$ on désigne par $q_{n}$ l'application de $Y^{n+1}$ sur $Y^{n+1} / \Gamma$. Soit d'autre part $G$ un groupe topologique. Dans l'ensemble $H^{1}(X, G)$ des classes d'espaces fibrés principaux de base $X$ et de groupe $G$, on va définir une filtration

$$
H^{1}(X, \mathbf{G})=Q_{0} \supset Q_{1} \supset Q_{2}
$$

analogue à celle qui a été définie plus haut pour la cohomologie singulière à coefficients constants.

Les applications $\mathbf{d}_{1, i}: Y^{2} / \Gamma \rightarrow Y / \Gamma$ et $\mathbf{d}_{2, j}: Y^{3} / \Gamma \rightarrow Y^{2} / \Gamma(i=0,1, j=0,1,2)$ vérifient les relations 


$$
\begin{aligned}
& d_{1,0} d_{2,0}=d_{1,0} d_{2,1} \\
& d_{1,0} d_{2,2}=d_{1,1} d_{2,0} \\
& d_{1,1} d_{2,1}=d_{1,1} d_{2,2} .
\end{aligned}
$$

Pour tout espace fibré principal de base $X=Y / \Gamma$ et de groupe $G$, ces applications définissent des fibrés principaux images réciproques de base $Y^{2} / \Gamma$ :

$$
P_{0}=P \mathbf{d}_{1,0}, \quad P_{1}=P \mathbf{d}_{1,1} .
$$

L'espace fibré $P$ sera appelé un espace fibré pré-associé à $Y$ lorsque $P_{0}$ et $P_{1}$ sont isomorphes. Le sous-ensemble $Q_{1}$ de $H^{1}(X, G)$ sera l'ensemble des classes de fibrés pré-associés à $Y$.

Lemme 1. Pour qu'un espace fibré principal $P$ de base $X$ soit pré-associé à $Y$, il faut qu'il soit trivialisé par la projection $q_{0}: Y \rightarrow X$.

En effet, si $P$ est préassocié à $Y, P q_{0} d_{1,0}$ et $P q_{0} d_{1,1}$ sont deux espaces fibrés isomorphes de base $Y^{2}$. Soit $b$ un point de $Y$ et soit $f$ l'application continue de $Y$ dans $Y^{2}$ définie par $f(y)=(b, y)$. L'espace fibré $P q_{0}=P q_{0} d_{1,0} f$ est isomorphe à l'espace fibré $P q_{0} d_{1,1} f$ qui est trivial puisque l'application $d_{1,1} f$ est constante.

Considérons maintenant les espaces fibrés images réciproques de $P_{1}$ et $P_{0}$ par les applications $\mathbf{d}_{2, j}: Y^{3} / \Gamma \rightarrow Y^{2} / \Gamma$ :

$$
\begin{array}{lll}
P_{0,0}=P_{0} \mathbf{d}_{2,0}, & P_{0,1}=P_{0} \mathbf{d}_{2,1}, & P_{0,2}=P_{0} \mathbf{d}_{2,2} \\
P_{1,0}=P_{1} \mathbf{d}_{2,0}, & P_{1,1}=P_{1} \mathbf{d}_{2,1}, & P_{1,2}=P_{1} \mathbf{d}_{2,2} .
\end{array}
$$

Les relations (1) montrent qu'il existe des isomorphismes canoniques

$$
P_{0,1} \stackrel{\lambda_{1}}{\longrightarrow} P_{0,0}, \quad P_{1,0} \stackrel{\lambda_{2}}{\longrightarrow} P_{0,2}, \quad P_{1,1} \stackrel{\lambda_{3}}{\longrightarrow} P_{1,2} .
$$

Supposons que $P$ soit pré-associé à $Y$. Tout isomorphisme $h$ de $P_{0}$ sur $P_{1}$ définit un isomorphisme $h_{j}$ de $P_{0, j}$ sur $P_{1, j}(j=0,1,2)$. Ainsi $h$ définit un diagramme :

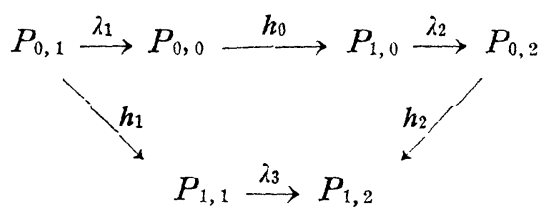

On dira que l'espace fibré $P$ est associé à $Y$ lorsque l'isomorphisme $h$ peut être choisi de telle sorte que ce diagramme soit commutatif. L'ensemble $Q_{2} \subset H^{1}(X, \mathbf{G})$ 
sera l'ensemble des classes de fibrés associés à $Y$. Cette terminologie se trouvera justifiée dans le paragraphe suivant.

4. Facteurs des espaces fibrés associés et pré-associés. Les notations restant celles du paragraphe précédent, soit $F(Y, G)$ l'ensemble des facteurs sur $Y$ à valeurs dans $G$, c'est à dire l'ensemble des applications continues $k$ de $Y \times \Gamma$ dans $G$ telles que

$$
k(y, s) k(y s, t)=k(y, s t)
$$

quels que soient $y \in Y, s, t \in \Gamma$. Tout facteur $k$ définit un espace fibré principal de base $X$ et de groupe $G$ : c'est le quotient de $Y \times G$ par $\Gamma$, les opérations de $\Gamma$ dans $Y \times G$ étant définies par $(y, a) s=(y s, a k(y, s))$ quels que soient $y \in Y$, $s \in \Gamma$ et $a \in G$. En associant à tout facteur $k \in F(Y, G)$ la classe [k] de cet espace fibré, on définit une application canonique de $F(Y, G)$ dans $H^{1}(X, \mathbf{G})$. L'image de cette application est l'ensemble des classes de fibrés trivialisés par la projection $q_{0}: Y \rightarrow X$, autrement dit c'est le "noyau" de l'application $H^{1}(X, \mathbf{G})$ $\rightarrow H^{1}(Y, \mathbf{G})$ définie par $q_{0}$. Pour que deux facteurs $k$ et $k^{\prime} \in F(Y, G)$ aient même image dans $H^{1}(X, \mathbf{G})$, il faut et il suffit qu'ils soient équivalents en ce sens qu'il existe une application continue $r: Y \rightarrow G$ telle que

$$
r(y) k^{\prime}(y, s)=k(y, s) r(y s)
$$

quels que soient $y \in Y$ et $s \in \Gamma$.

ThÉoRème 5. Soit $k$ un facteur sur $Y$ à valeurs dans $G$. Pour que les espaces fibrés de classe [k] soient pré-associés à $Y$, il faut et il suffit qu'il existe une application continue $g$ de $Y^{2}$ dans $G$ telle que

$$
\boldsymbol{g}\left(y, y^{\prime}\right) k\left(y^{\prime}, s\right)=k(y, s) g\left(y s, y^{\prime} s\right)
$$

quels que soient $y, y^{\prime} \in Y$ et $s \in T$.

Soit en effet $P$ un espace fibré de classe $[k]$. Il existe une application continue $r$ de $Y$ dans $P$ compatible avec les projections sur $X$ telle que $r(y s)$ $=r(y) k(y, s)$ pour tout $y \in Y$ et tout $s \in \Gamma$. Soit $r_{i}(i=0,1)$ l'application continue de $Y^{2}$ dans l'espace fibré $P_{i}=P \mathbf{d}_{1, i}$ définie par

$$
r_{i}\left(y, y^{\prime}\right)=\left(q_{1}\left(y, y^{\prime}\right), r d_{1, i}\left(y, y^{\prime}\right)\right) .
$$

Les applications $r_{i}$ sont compatibles avec les projections sur $Y^{2} / l^{\prime}$ et vérifient 
les relations :

$$
r_{i}\left(y s, y^{\prime} s\right)=r_{i}\left(y, y^{\prime}\right) k_{i}\left(y, y^{\prime}, s\right)
$$

où $k_{i}\left(y, y^{\prime}, s\right)=\bar{k}\left(d_{1, i}\left(y, y^{\prime}\right), s\right)$ quels que soient $y, y^{\prime} \in Y$ et $s \in \Gamma$. Soit $h$ un isomorphisme de $P_{0}$ sur $P_{1}$. L'application $g$ de $Y^{2}$ dans $G$ définie par

$$
h\left(r_{0}\left(y, y^{\prime}\right)\right)=r_{1}\left(y, y^{\prime}\right) g\left(y, y^{\prime}\right)
$$

est continue et vérifie la relation

$$
g\left(y, y^{\prime}\right) k_{0}\left(y, y^{\prime}, s\right)=k_{1}\left(y, y^{\prime}, s\right) g\left(y s, y^{\prime} s\right)
$$

c'est à dire (PA). Réciproquement, on voit facilement que si $g$ est une application continue de $Y^{2}$ dans $G$ qui vérifie la condition (PA) alors il existe un isomorphisme $h: P_{0} \rightarrow P_{1}$ (et un seul) tel que $h\left(r_{0}\left(y, y^{\prime}\right)\right)=r_{1}\left(y, y^{\prime}\right) g\left(y, y^{\prime}\right)$ quels que soient $y, y^{\prime} \in Y$.

Théorème 6. Soit $k$ un facteur sur $Y$ à valeurs dans $G$. Pour que les espaces fibrés de classe [k] soient associés à $Y$, il faut et il suffit qu'il existe une application continue $g: Y^{2} \rightarrow G$ telle que

$$
\begin{aligned}
& g\left(y, y^{\prime}\right) k\left(y^{\prime}, s\right)=k(y, s) g\left(y s, y^{\prime} s\right) \\
& g\left(y, y^{\prime}\right) g\left(y^{\prime}, y^{\prime \prime}\right)=g\left(y, y^{\prime \prime}\right)
\end{aligned}
$$

quels que soient $y, y^{\prime}, y^{\prime \prime} \in Y$ et $s \in \Gamma$.

Soit en effet $P$ un espace fibré de classe $[k]$ et $r$ une application continue de $Y$ dans $P$ telle que $r(y s)=r(y) k(y, s)$ pour tout $y \in Y$ et tout $s \in \Gamma$. Posons comme plus haut $r_{i}\left(y, y^{\prime}\right)=\left(q_{1}\left(y, y^{\prime}\right), r d_{1, i}\left(y, y^{\prime}\right)\right)$ et $k_{i}\left(y, y^{\prime}, s\right)=k\left(d_{1, i}\left(y, y^{\prime}\right), s\right)$ où $i=0,1$. Pour $i=0,1$ et $j=0,1,2$ on définit une application continue $r_{i, j}$ de $Y^{3}$ dans $P_{i, j}=P_{i} \mathbf{d}_{2, j}$ en posant:

$$
r_{i, j}\left(y, y^{\prime}, y^{\prime \prime}\right)=\left(q_{2}\left(y, y^{\prime}, y^{\prime \prime}\right), r_{i} d_{2, j}\left(y, y^{\prime}, y^{\prime \prime}\right)\right)
$$

quels que soient $y, y^{\prime}, y^{\prime \prime}$. Le diagramme constitué par les applications $\boldsymbol{r}_{i, j}$ et les isomorphismes canoniques $P_{0,1} \stackrel{\lambda_{1}}{\longrightarrow} P_{0,0}, P_{1,0} \stackrel{\lambda_{2}}{\longrightarrow} P_{0,2}, P_{1,1} \stackrel{\lambda_{3}}{\longrightarrow} P_{1,2}$ est commutatif. Supposons maintenant que $P$ soit préassocié à $Y$. Soit $h$ un isomorphisme de $P_{0}$ sur $P_{1}$ et soit $h_{j}$ l'isomorphisme de $P_{0, j}$ sur $P_{1, j}$ défini par $h$. Soit $g$ l'application continue de $Y^{2}$ dans $G$ définie par la condition $h r_{0}\left(y, y^{\prime}\right)$ $=r_{1}\left(y, y^{\prime}\right) g\left(y, y^{\prime}\right)$ quels que soient $y, y^{\prime} \in Y$. On a

$$
h_{j} r_{0, i}\left(y, y^{\prime}, y^{\prime \prime}\right)=r_{1}, j\left(y, y^{\prime}, y^{\prime \prime}\right) g\left(d_{2, j}\left(y, y^{\prime}, y^{\prime \prime}\right)\right)
$$


et par suite

$$
\begin{aligned}
& \lambda_{3} h_{1} r_{0,1}\left(y, y^{\prime}, y^{\prime \prime}\right)=r_{1,2}\left(y, y^{\prime}, y^{\prime \prime}\right) g\left(y, y^{\prime \prime}\right) \\
& h_{2} \lambda_{2} h_{0} \lambda_{1} r_{0,1}\left(y, y^{\prime}, y^{\prime \prime}\right)=r_{1,2}\left(y, y^{\prime}, y^{\prime \prime}\right) g\left(y, y^{\prime}\right) g\left(y^{\prime}, y^{\prime \prime}\right)
\end{aligned}
$$

quels que soient $y, y^{\prime}, y^{\prime \prime} \quad Y$. Ceci montre que, pour que le diagramme $(H)$ défini par $h$ soit commutatif, il faut et il suffit que $g$ vérifie, en plus de la condition ( $\mathrm{PA})$ la condition $(\mathrm{A})$. Le Théorème en résulte aussitôt.

THÉoRÈme 7. Soit $k$ un facteur sur $Y$ à valeurs dans $G$. Pour qu'il existe une application continue $g$ de $Y^{2}$ dans $G$ vérifiant les conditions $(P A)$ et $(A)$ du Théorème 6, il faut et il suffit que $k$ soit équivalent à un homomorphisme continu de $I$ dans $G$.

En effet, si $k$ est un homomorphisme continu de $\Gamma$ dans $G$, l'application constante $g$ de $Y^{2}$ sur l'élément neutre de $G$ vérifie les conditions (PA) et (A). D'autre part, si $g$ est une application continue de $Y^{2}$ dans $G$ vérifiant ces conditions, alors si $b \in Y, k$ est équivalent au facteur $k^{\prime}$ défini par $k^{\prime}(y, s)$ $=g(y, b)^{-1} k(y, s) g(y s, b)$. Compte tenu de $(A)$, on a $k^{\prime}(y, s)=g(b, b)^{-1}$ $g(y, b)^{-1} k(y, s) g(y s, b s) g(b s, b)=g(b, b)^{-1} k(b, s) g(b s, b)=k^{\prime}(b, s)$, ce qui montre que $k^{\prime}$ est un homomorphisme de $I$ dans $G$.

Avec les Théorèmes 6 et 7 , on voit que les espaces fibrés de groupe $G$ associés à $Y$ se déduisent de $Y$ par le procédé habituel d'extension du groupe de structure et sont donc des espaces fibrés associés à $Y$ au sens ordinaire.

Exemple. Supposons que $Y$ soit le revêtement universel d'un espace topologique $X$ connexe localement compact et dénombrable à l'infini, $\Gamma$ étant le groupe des automorphismes de $Y$. Soit $0 \longrightarrow \Delta \longrightarrow \mathbf{R}^{n} \stackrel{\rho}{\longrightarrow} G \longrightarrow 0$ une suite exacte de groupes abéliens dans laquelle $\Delta$ est un sous-groupe discret de $\mathbf{R}^{n}$. Puisque $Y$ est simplement connexe, si $k$ est un facteur sur $Y$ à valeurs dans $G$, il existe une application continue $h$ de $Y \times \Gamma$ dans $R^{n}$ telle que $k(y, s)=\rho h(y, s)$ pour tout $y \in Y$ et tout $s \in I$. On a $h(y, s)+h(y s, t)-h(y, s t) \in \Delta$ quels que soient $y \in Y$ et $s, t \in \Gamma$. Par conséquent, $m\left(y, y^{\prime}, s\right)=h(y, s)-h\left(y^{\prime}, s\right.$.) est un facteur sur $Y^{2}$ (considéré comme espace fibré principal de groupe $T$ ) à valeurs dans $\mathbf{R}^{n}$. Comme tout espace fibré principal de base $Y^{2} / I$ de groupe $\mathbf{R}^{n}$ est trivial, ce facteur $m$ est équivalent au facteur constant nul. Autrement dit, il existe une application continue $r: Y^{2} \rightarrow \mathbf{R}^{n}$ telle que $m\left(y, y^{\prime}, s\right)=r\left(y, y^{\prime}\right)$ 
$-r\left(y s, y^{\prime} s\right)$ quels que soient $y, y^{\prime} \in Y$ et $s \in \Gamma$. Posons $g\left(y, y^{\prime}\right)=\rho r\left(y, y^{\prime}\right)$. On a $g\left(y, y^{\prime}\right) k\left(y^{\prime}, s\right)=\rho\left(r\left(y, y^{\prime}\right)+h\left(y^{\prime}, s\right)\right)=\rho\left(r\left(y s, y^{\prime} s\right)+h(y, s)\right)=k(y, s) g\left(y s, y^{\prime} s\right)$. Par conséquent tout espace fibré principal de groupe $G$ qui est trivialisé par $Y \rightarrow X$ est pré-associé à $Y$.

5. Restriction du groupe de structure pour les espaces fibrés pré-associés. On conserve les notations des paragraphes 3 et 4 .

Lemme 2. Soit $k$ un facteur sur $Y$ à valeurs dans $G$ tel que les espaces fibrés de classe $[k]$ soient pré-associés à $Y$. Il existe une application continue $g$ de $Y^{2}$ dans $G$ qui vérifie, en plus de la condition ( $P A$ ) la condition

$$
g(y, y)=e \quad(\text { élément neutre de } G)
$$

pour tout $y \in Y$.

En effet, si $g^{\prime}$ est une application continue de $Y^{2}$ dans $G$ vérifiant la condition (PA), alors

$$
g^{\prime}\left(y, y^{\prime}\right) g^{\prime}\left(y^{\prime}, y^{\prime}\right)^{-1} k\left(y^{\prime}, s\right)=k(y, s) g^{\prime}\left(y s, y^{\prime} s\right) g^{\prime}\left(y^{\prime} s, y^{\prime} s\right)^{-1},
$$

donc, en posant $g\left(y, y^{\prime}\right)=g^{\prime}\left(y, y^{\prime}\right) g^{\prime}\left(y^{\prime}, y^{\prime}\right)^{-1}$, on obtient une application $g$ qui vérifie les conditions $(\mathrm{PA})$ et $(\mathrm{N})$.

Dans ce qui suit, on suppose que $k$ est un facteur sur $Y$ à valeurs dans $G$ et que $g$ est une application vérifiant les conditions (PA) et $(\mathrm{N})$. Pour tout $b \in Y$, on notera $G(b)$ le sous-groupe de $G$ engendré par les éléments de la forme

$$
g(b, y) g\left(y, y^{\prime}\right) g\left(b, y^{\prime}\right)^{-1}
$$

où $y, y^{\prime} \in Y$. Quels que soient $b, b^{\prime} \in Y$, on a $G(b)=g\left(b, b^{\prime}\right) G\left(b^{\prime}\right) g\left(b, b^{\prime}\right)^{-1}$. D'autre part, pour tout $s \in I ; k(b, s) g(b, b s)^{-1}$ est dans le normalisateur de $G(b)$ dans $G$. On désigne par $H(b)$ le sous-groupe de $G$ engendré par $G(b)$ et les éléments de la forme $k(b, s) g(b, b s)^{-1}$ avec $s \in \Gamma$. On a $H(b)$ $=g\left(b, b^{\prime}\right) H\left(b^{\prime}\right) g\left(b, b^{\prime}\right)^{-1}$ quels que soient $b, b^{\prime} \in Y$. L'application $s \rightarrow k(b, s) g(b, b s)^{-1}$ composée avec l'homomorphisme canonique de $H(b)$ sur $H(b) / G(b)$ donne un homomorphisme surjectif de $I$ sur $H(b) / G(b)$.

THÉORÈme 8. Le groupe de structure des espaces fibrés préassociés à $Y$ de classe [k] peut être restreint au sous-groupe $H(b)$ de $G$ et l'espace fibré de groupe $H(b)$ obtenu est encore trivialisé par l'application $Y \rightarrow X$. 
En effet, le facteur $k$ est équivalent au facteur $k^{\prime}$ défini par $k^{\prime}(y, s)$ $=g(b, s) k(y, s) g(b, y s)^{-1}=k(b, s) g(b s, y s) g(b, y s)^{-1}=k(b, s) g(b, b s)^{-1} v$, avec $v \in G(b)$, c'est à dire que $k$ est équivalent à un facteur à valeurs dans le sousgroupe $H(b)$ de $G$.

6. Espaces fibrés pré-associés et formes de connexions. Dans ce paragraphe, on suppose que $Y$ est un espace fibré principal differentiable de base $X$ et de groupe $G$; on suppose de plus que $G$ est un groupe de Lie. Soit $P$ un espace fibré différentiable de base $X$ et de groupe $G$ trivialisé par la projection $q: Y \rightarrow X$ et soit $r$ une application différentiable de $Y$ dans $P$ compatible avec les projections sur $X$. On désigne par $k$ le facteur différentiable sur $Y$, à valeurs dans $G$, tel que $r(y s)=r(y) k(y, s)$ quels que soient $y \in Y$ et $s \in \Gamma$. Pour toute forme de connexion $r$ sur $P_{,}^{2)}$ la forme $\omega=\gamma r$, image réciproque de $r$ par $r$, est une forme différentielle de degré 1 sur $Y$, à valeurs dans l'algèbre de Lie $\mathbf{g}$ de $G$, telle que

$$
k(y, s) \omega(d y s+y d s)-\omega(d y) k(y, s)=k(d y, s)+k(y, d s)
$$

quels que soient les vecteurs dy d'origine $y \in Y$ et $d s$ d'origine $s \in \Gamma$. Réciproquement, soit $\omega$ une forme différentielle de degré 1 sur $Y$ à valeurs dans $\mathbf{g}$ qui vérifie la condition $(\mathrm{C})$. Sur $Y \times G$, on considère la forme $\eta$ telle que

$$
\eta(d y, d a)=a \omega(d y) a^{-1}-d a a^{-1}
$$

quels que soient les vecteurs dy d'origine $y \in Y$ et $d a$ d'origine $a \in G$. On vérifie facilement qu'il existe sur $P$ une forme $\gamma$ et une seule telle que $\eta$ soit image réciproque de $r$ par l'application $(y, a) \rightarrow r(y) a^{-1}$ de $Y \times G$ sur $P$. Et cette forme $r$ est une forme de connexion sur $P$. Ainsi, l'application $r \rightarrow r r$ est une bijection de l'ensemble des formes de connexion sur $P$ sur l'ensemble des formes différentielles sur $Y$ à valeurs dans $\mathrm{g}$ qui vérifient la condition (C).

La condition (C) est équivalente aux deux conditions:

$$
k(y, s) \omega(d y, s)-\omega(d y) k(y, s)=k(d y, s)
$$

2) Pour la notion de forme de connexion, voir par exemple K. Nomizu [6]. On utilise ici les conventions d'écriture suivantes. Pour toute application différentiable $f$, on. désigne par $f(d y)$ le vecteur image d'un vecteur $d y$ par l'application "dérivée" de $f$. Si $(y, s)$ $\in Y \times \Gamma$, on désigne par $(d y, s)$ le vecteur de la variété $Y \times \Gamma$ image du vecteur $d y$ de $Y$ par l'application $z \rightarrow(z, s)$. De meme $(y, d s)$ est l'image de $d s$ par l'application $t \rightarrow(y, d t)$. On pose $(d y, d s)=(d y, s)+(y, d s)$. L'image de $(d y, s)($ resp. $(y, d s))$ par l'application $\mathrm{Y} \times \mathrm{Y}$ $\rightarrow Y$ qui est définie par les opérations de $\Gamma$ dans $Y$ se note $d y s$ (resp. $y d s$ ). 
pour tout $s \in \Gamma$ et tout vecteur dy d'origine $y \in Y$,

$$
k(y, s) \omega(y d s)=k(y, d s)
$$

pour tout $y \in Y$ et tout vecteur ds d'origine $s \in I$.

Supposons maintenant que $P$ soit un espace fibré pré-associé à $Y$. Il existe alors une application differentiable $g$ de $Y^{2} \rightarrow G$ telle que les conditions (PA) et (N) soient satisfaites. De (PA) on déduit que

$$
g\left(y, d y^{\prime}\right) k\left(y^{\prime}, s\right)+g\left(y, y^{\prime}\right) k\left(d y^{\prime}, s\right)=k(y, s) g\left(y s,\left(d y^{\prime}\right) s\right)
$$

pour tout $s \in \Gamma, y \in Y$ et tout vecteur $d y^{\prime}$ d'origine $y^{\prime} \in Y$. Pour $y=y^{\prime}$ on obtient $g(y, d y) k(y, s)+k(d y, s)=k(y, s) g(y s,(d y) s)$ ce qui signifie que la forme différentielle $\omega$ sur $Y$ définie par $\omega(d y)=g(y, d y)$ vérifie la condition (C.1).

Si le groupe $I$ ' est discret, la relation (C.2) est trivialement vérifiée. Dans ce cas, le choix d'une application $g: Y^{2} \rightarrow G$ vérifiant les conditions (PA) et (N) déterminera donc une forme de connexion sur l'espace fibré principal $P$. Les groupes d'holonomie de cette connexion sont liés aux groupes $H(b)$ et $G(b)$ définis par $g(\mathrm{cf} . \S 5)$. D'une manière précise, si $r$ désigne l'application différentiable de $Y$ dans $P$ telle que $r(y s)=r(y) k(b, s)$, et si $r$ est la forme de connexion sur $P$ telle que $g(y, d y)=\gamma r(d y)$, alors, pour tout point $b \in Y$ le groupe d'holonomie de $r$ au point $r(b)$ est contenu dans $H(b)$ et le groupe d'holonomie restreinte de $r$ au point $r(b)$ est contenu dans $G(b)$. Soit en effet $c(t)$ un lacet différentiable d'origine et d'extrémité $g(b)$ dans $X$ et soit $y(t)$ le chemin d'origine $b$ dans $Y$ tel que $q p(t)=c(t)(t \in I=[0,1])$. Le chemin $r y(t)$ est un relèvement de $c(t)$ dans $P$ ayant pour origine $r(b)$. Le relèvement de $c(t)$ dans $P$ ayant pour origine $r(b)$ qui est intégral pour la forme de connexion $r$ s'écrit $(r y(t)) \theta(t)$ où $\theta$ est un chemin d'origine $e$ dans $G$. Si $s$ est l'élément de $\Gamma$ défini par $y(1)=b s$, alors $r y(1)=r(b) k(b, s)$ est l'élément du groupe d'holonomie au point $r(b)$ défini par le lacet $c(t)$ et $k(b, s) \theta(1)$. On a $r((r y(d t)) \theta(t)$ $+(r y(t)) \theta(d t))=0$ pour tout vecteur $d t$ d'origine $t \in I$, par conséquent, $\theta(t)^{-1}(\gamma r y(d t)) \theta(t)+\theta(t)^{-1} \theta(d t)=0$. Puisque $\quad r r(d y)=g(y, d y)$, ceci donne $g(y(t), y(d t))=-\theta(d t) \theta(t)^{-1}$. D'autre part, d'après la définition de $G(b)$ (cf. $\S 5$ ) l'application $u: I^{2} \rightarrow G$ définie par

$$
g(b, y(t)) g\left(y(t), y\left(t^{\prime}\right)\right)=u\left(t, t^{\prime}\right) g\left(b, y\left(t^{\prime}\right)\right)
$$

a ses valeurs dans la composante connexe par arcs de l'élément neutre de $G(b)$ 
qui est un sous-groupe de Lie de $G([3])$. En dérivant la relation précédente par rapport à $t^{\prime}$ puis en posant $t=t^{\prime}$, on obtient

$$
g(b, y(t)) g(y(t), y(d t))=u(t, d t) g(b, y(t))+g(b, y(d t))
$$

pour tout vecteur $d t$ d'origine $t \in I$. Il en résulte que $u(t, d t)=-f(d t) f(t)^{-1}$ où $f(t)=g(b, y(t)) \theta(t)$. Par conséquent, $f(t) \in G(b)$ pour tout $t \in I$ et en particulier $f(1)=g(b, y(1)) \theta(1)=g(b, b s) \theta(1) \in G(b)$. On a donc $k(b, s) \theta(1)$ $=k(b, s) g(b, b s)^{-1} g(b, b s) \theta(1) \in H(b)$. Si le lacet $c(t)$ est homotope à 0 dans $X$, alors $y(1)=b$, donc $s=e$ et $k(b, s) \theta(1)=\theta(1)=f(1) \in G(b)$, ce qui démontre l'assertion.

7. Cas des espaces fibrés holomorphes. Soient $\Gamma$ un groupe de Lie complexe et $Y$ un espace fibré holomorphe de groupe $\Gamma$ et de base $X$. On définit comme au paragraphe 3 les espaces fibrés principaux holomorphes de base $X$ associés et pré-associés à $Y$, les isomorphismes intervenant dans la définition étant alors des isomorphismes holomorphes. Les facteurs étant maintenant des applications holomorphes de $Y$ dans un groupe de Lie complexe $G$, les Théorèmes 5,6 ainsi que le Lemme 2 subsistent en $y$ remplaçant le mot "continu" par "holomorphe". La condition, pour un espace fibré principal de base $X$ d'être pré-associé à $Y$ devient très restrictive dans le cadre holomorphe. Compte tenu du paragraphe 6 , on voit par exemple que, si $Y$ est un revêtement galoisien de $X$, tout espace fibré principal holomorphe pré-associé à $Y$ possède une connexion holomorphe ([1]).

Dans le cas où $Y=C^{n}$ et où $\Gamma$ est un sous-groupe discret opérant par translations dans $C^{n}$, tout espace fibré principal holomorphe $P$ de base $X=Y / \Gamma$ qui possède une connexion holomorphe est préassocié à $Y$. En effet, puisque $P$ est trivialisé par $Y \rightarrow X$ il existe une application holomorphe $r$ de $Y$ dans $P$ compatible avec les projections sur $X$. Soit $k$ le facteur holomorphe sur $Y$ défini par $r(y+s)=r(y) k(y, s)$ pour $y \in Y$ et $s \in \Gamma$. Soit $\gamma$ une forme de connexion holomorphe sur $P$. Pour tout $\left(y, y^{\prime}\right) \in Y^{2}$ on désigne par $\theta\left(y, y^{\prime}, \quad\right)$ l'application différentiable de l'intervalle $I=[0,1]$ dans $G$ telle que le chemin $t \rightarrow r\left(y+t\left(y^{\prime}-y\right)\right) \theta\left(y, y^{\prime}, t\right)$ soit un chemin intégral d'origine $r(y)$ pour la forme de connexion $r$. Si $\omega=r r$, on a donc $\theta\left(y, y^{\prime}, 0\right)=e$ et $\theta\left(y, y^{\prime}, d t\right)$ $=-\omega\left(y+d t\left(y^{\prime}-y\right)\right) \theta\left(y, y^{\prime}, t\right)$ pour tout vecteur $d t$ d'origine $t$ dans $I$. Puisque $\gamma$ est holomorphe, il en est de même de la forme $\omega$ sur $Y$ et il en résulte que. 
pour chaque valeur de $t$, l'application $\left(y, y^{\prime}\right) \rightarrow \theta\left(y, y^{\prime}, t\right)$ est une application holomorphe de $\dot{Y}^{2}$ dans $G$. Quel que soit $s \in \Gamma, t \rightarrow r\left(y+s+t\left(y^{\prime}-y\right)\right) \theta(y+s$, $\left.y^{\prime}+s, t\right)=r\left(y+t\left(y^{\prime}-y\right)\right) k(y, s) \theta\left(y+s, y^{\prime}+s, t\right)$ et $t \rightarrow r\left(y+t\left(y^{\prime}-y\right)\right) \theta\left(y, y^{\prime}, t\right)$ sont deux chemins intégraux dans $P$ qui se projettent suivant le même chemin sur $X$ et qui ont respectivement pour origines $r(y+s)=r(y) k(y, s)$ et $r(y)$. Par suite, $k\left(y+t\left(y^{\prime}-y\right), s\right) \theta\left(y+s, y^{\prime}+s, t\right)=\theta\left(y, y^{\prime}, t\right) k(y, s)$ quels que soient $y, y^{\prime} \in Y, \quad s \in \Gamma$ et $t \in I$. Pour $t=1$, on a donc $k\left(y^{\prime}, s\right) \theta\left(y+s, y^{\prime}+s, 1\right)$ $=\theta\left(y, y^{\prime}, 1\right) k(y, s)$, c'est à dire que l'application holomorphe $g$ de $Y^{2}$ dans $G$ définie par $g\left(y, y^{\prime}\right)=\theta\left(y, y^{\prime}, 1\right)^{-1}$ vérifie la condition (PA).

Dans le cas où $n>1$ et où $X$ est compact, S. Murakami a démontré l'existence d'espaces fibrés holomorphes de base $X$ qui possèdent des connexions holomorphes mais ne possèdent pas de connexion holomorphe intégrable [5]. Il en résulte que, pour $n>1$, il existe des espaces fibrés holomorphes préassociés à $Y$ qui ne sont pas associés à $Y$.

\section{REFÉRENCES}

[1] A. M. F. Atiyah, Complex analytic connections in Fibre Bundle, Trans. of the Amer. Math. Soc. vol. 85 (1957), pp. 181-207.

[2] J. L. Koszul, Multiplicateurs et classes caracteristiques, Trans. of Amer. Math. Soc. 89 (1958), pp. 256-267.

[3] H. M. Yamabe, On arc-wise connected subgroups of a Lie group, Osaka Math. J. vol. 2 (1957), pp. 13-14.

[4] J. Milnor, Construction of universal bundles, II, Ann. of Math. vol. 63 (1956), pp. 430-436.

[5] S. Murakami, Sur certains espaces fibres principaux holomorphes admettant des connexions holomorphes, Osaka Math. J. vol. 11 (1959), pp. 43-62.

[6] K. Nomizu, Lie groups and differential Geometry, Math. Soc. of Japan 2 (1956).

\section{Université de Strasbourg}

